\title{
Intellectual Developmental Disability Bio-Banking: Public Support from the European Union
}

\author{
Carlos Moedas
}

200 Rue de la Loi, B-1049 Brussels, Belgium

\begin{abstract}
Supporting health research has been a long-standing commitment for the European Union (EU). Over several Framework Programmes for Research and Innovation, the EU contributed to advancing scientific knowledge on intellectual disability and addressing the unmet needs of affected people, their families and carers. Bio-banking is instrumental to such research. This article presents the various streams through which the EU has helped developments in this area and highlights opportunities for the future.
\end{abstract}

Keywords: Research and innovation, Horizon 2020, Framework Programme, European Commission, intellectual disability.

\section{INTRODUCTION}

Our brain is the source of emotions, behaviour and intellectual capacities, enabling us to socially interact. Understanding how the human brain works is a real challenge. Such knowledge would open many doors, notably by providing the key to brain malfunction and bringing solutions to restore health, wellbeing and personality.

Brain disorders place a heavy burden on society. In 2010 alone, the total cost of brain disorders in $27 \mathrm{EU}$ member states and three countries associated to the Seventh Framework Programme for Research and Technological Development (FP7) was estimated to be around EUR 800 billion [1].

Amongst brain disorders, intellectual disability represents an important burden worldwide with an overall prevalence estimated at more than 10/1000 population [2]. For some 4.2 million people in Europe [3], as well as their families and carers, it translates to a reduced quality of life. The economic impact is also high: the yearly cost of mental retardation in $27 \mathrm{EU}$ member states and three countries associated to FP7 was estimated around EUR 43.3 billion in 2010 [1].

The challenge posed by intellectual disability is multifaceted, encompassing several sectors such as care, education, employment and social inclusion. This means that solutions should be multidisciplinary, spanning the whole range of actions from research to improve knowledge to translating that knowledge into better care and social inclusion of people with intellectual disability. The European Commission is at

*Address correspondence to this author at the 200 Rue de la Loi, B-1049 Brussels, Belgium; Tel: +32 229-55373; E-mail: carlos.moedas@ec.europa.eu the forefront of promoting multidisciplinary and international research in these areas.

FP7 invested EUR 2.9 billion in research on the brain and brain diseases between 2007 and 2013 . Research supported included research on intellectual disability (EUR 138 million) and research on autism spectrum disorders (ASD, EUR 117 million). The World Health Organization estimates that around $50 \%$ of persons with ASD also suffer from an intellectual disability. Such research was geared towards a better understanding of the mechanisms underlying autism and intellectual disability and the generation of knowledge and tools for earlier and better detection, better care, including the development of innovative drugs, and improved social inclusion of affected patients.

Bio-banks are key resources underpinning research, allowing light to be shed on the genetic, physiological and environmental determinants underlying the development of diseases. For diseasespecific bio-banks, biomaterial such as DNA, RNA, serum, whole blood, cells or tissues is to be accompanied by genetic, clinical, environmental and life-style information on individuals, as relevant to the type of research to be performed. Such clinical biobanks are essential tools to identify biomarkers for prognosis, diagnosis, disease prediction and advance the development of a stratified, targeted medicine.

\section{SUPPORT FROM THE EUROPEAN UNION TO INTELLECTUAL DISABILITY}

Most support from the European Union to intellectual disability bio-banking has taken place through collaborative research projects, within which the collection and/or analysis of samples is embedded. Some examples follow: 


\section{Childhood Therapeutic Interventions in Compulsive Syndromes: TACTICS (FP7-SECURITY)}

The FP7 project TACTICS [4] focuses on adolescent and childhood therapeutic interventions in compulsive syndromes, including stereotypical compulsive behaviour of ASD. The project is aimed at better understanding the neural, genetic and molecular factors involved in the pathogenesis of compulsivity through structural neuroimaging, neurochemistry, behaviour, genetics, proteomics studies and machine learning tools in paediatric clinical populations. The efficacy of pharmacological interventions will ultimately be tested in paediatric populations. The consortium identified an age-dependent biomarker signature in children and adolescents with ASD [5]. These deviations in age-dependent molecular trajectories provide further insight into the pathophysiology and progression of the disorder. Such results underline the importance of stratifying and analysing samples by age, especially in ASD and potentially in other developmental disorders. The results may contribute to a better classification of ASD individuals, and as a consequence, to improved treatment and prognosis.

\section{Neurotoxic Compounds: The Project DENAMIC}

The increased incidence of learning and developmental disorders in children has led to serious concern worldwide. However we currently lack the evidence-base for identifying potential neurotoxic compounds and for managing the associated risks. This is why the European Union is supporting the project DENAMIC [6], which investigates neurotoxic effects of low-concentration mixtures of pesticides and a number of common environmental pollutants in children. The project is developing tools and methods for screening neurotoxic effects and focuses on (subclinical) effects on learning (cognitive skills) and developmental disorders in children. Biomarkers for developmental neurotoxicity are being investigated, for which bio-banking activities are crucial. Genomic, proteomic and metabolomics markers are to be validated, in relation with behaviour, cognitive and motor functions and timing of exposure. First results indicate that the current use of insecticides during pregnancy in Europe, probably at low exposure levels and particularly spray application, is deleterious for the developing nervous system. More research is however needed to assess long-term neuropsychological delays and extended measures of the exposure, as well as possible modifiers of such toxicity [7]. Aware of the importance of risk factors as determinants of the development of diseases, the European Commission called for the submission of research applications in this area in its first call for proposals of the Horizon 2020 "Health, demographic change and wellbeing" societal challenge, SC1 $[8,9]$. Fourteen projects, including addressing environmental exposures, will be supported in the near future.

\section{Innovative Medicines Initiative (IMI)}

European efforts have also taken place within the Innovative Medicines Initiative (IMI), aimed at developing the next generation of medicines, vaccines and treatments through public-private partnering between academia, industry (large but also small and medium-size enterprises), patients and regulators. This mix of resources and expertise gives IMI its strength in speeding up the development of better and safer medicines for patients.

The work of IMI includes the EU-AIMS project [10], an alliance between academic institutions, the pharmaceutical industry, regulatory authorities and representatives of patients to research the genetic determinants and the biology of ASD and to develop better diagnosis and treatments for autism. For example, the consortium is gathering samples from people bearing certain mutations associated with ASD; this will pave the way for the generation of cell lines that can later be used to test treatments. The project includes a dedicated repository of samples and phenotypic and omics data acquired by the partners. Collaboration is established with other international biobank resources in order to harmonize standard operating procedures and the description of phenotypes, and provide unified access to data. The analysis of samples should lead to the identification of predictive and prognostic sets of markers, and to the characterisation of genetic mechanisms associated with ASD and autism-related behavioural traits. Ultimately, EU-AIMS is expected to improve the quality of life of people with ASD, while helping to curb the heavy economic and social costs of autism. EU-AIMS research already revealed that the father's age when a child is conceived is the biggest single contributor to the number of new mutations passed on to a child. The increase in the number of autism cases may hence be partly due to the fact that the average age of fathers at the time of conception is on the rise.

\section{ASPECTS TO CONSIDER IN EU COLLABORATIVE RESEARCH}

\section{Implementation of Common Standards}

The harmonisation of procedures and the implementation of common standards in a legally and 
ethically compliant way are important aspects to consider if one wants to reap the full benefits of EU collaborative research in a timely manner. The need for harmonisation, standardisation and quality control aspects of collected samples and data through the development of standard operating procedures is well recognised by the European Commission. For instance in 2012 the work programme of the FP7 Health Theme under the Cooperation Specific Programme called for the integration information from different omics platforms with clinical data in order to support the development of reference omics profiles for rare diseases, focusing on harmonising and standardising quality samples and data, and integrating existing resources whenever feasible. The selected project, RD-Connect [11], seeks to unite the multiple existing infrastructures and integrate the latest tools in order to create a robust and comprehensive combined biobanking, data analysis and patient registry platform for rare diseases, for use by researchers across the world.

\section{Privacy and Data Protection Requirements}

Variability with regards to privacy and data protection requirements exists among bio-banks in Europe, as it emerged from a 2010 study published by the European Commission Joint Research Centre [12]. Informed consent for approval of bio-bank-based research is almost ubiquitously required, but the actual consent requirements and related procedures vary widely among bio-banks, depending on national laws and guidelines. The European Commission proposed a major reform of the European data protection rules in 2012 [13], in order to address the changes that technological progress and globalisation brought in the way our data is collected, accessed and used. The proposal includes a single set of rules on data protection, valid across the EU and for all areas, not only health, not only research. Such rules should address the fragmentation of approaches in data protection. The European Commission is keen on a future European legislative framework that contributes to the right framework conditions for research in Europe in order to live up to the ambitions of the Europe 2020 strategy for smart, sustainable and inclusive growth [14]. In delivering a level-playing field in Europe with regard to the processing of data for research purposes, such a framework needs to provide favourable conditions for scientific research while guaranteeing high ethical standards and adequate protection of privacy rights. The reform is yet pending the approval by the European Council and the European Parliament $[15,16]$.

\section{Research Infrastructures}

Capitalising on existing successful bio-banking projects and networks appears essential, especially in times of resources constraints.

The European Union has for years dedicated specific funds to create new research infrastructures of pan-European interest in all fields of science and technology and to optimise the use and development of the best research infrastructures existing in Europe. The creation of the European Strategy Forum on Research Infrastructures (ESFRI) [17] in 2002 contributed to developing the scientific integration of European capacities and to strengthen its international outreach. Through ESFRI, national authorities can explore the best development and use of Research Infrastructures of pan-European relevance. In the field of biological and medical sciences, the Biobanking and Biomolecular Resources Research Infrastructure (BBMRI) [18] that started in February 2008 was one of the first European Research Infrastructure projects funded by the European Commission. BBMRI is a panEuropean distributed infrastructure of existing and new bio-banks and bio-molecular resource centres, with operational units in all participating Member States. It provides access to human biological samples essential for health research (e.g. blood, tissues, cells or DNA, associated with research and clinical data). It also comprises bio-molecular research and biocomputational tools for optimal exploitation of this resource. BBMRI is supporting bio-banks and networks of bio-banks, both for bio-banking samples and giving access to samples, in a wide variety of areas, including autism and diseases associated with mental retardation.

Since December 2013, BBMRI is operating through a legal and governance framework at European level, under a "European Research Infrastructure Consortium" (ERIC) legal structure. Twelve Member States are currently members of the BBMRI-ERIC $[19,20]$, with five observer countries and intergovernmental organisations. As indicated in its statutes, BBMRI-ERIC shall establish, operate and develop a pan-European distributed research infrastructure of bio-banks and bio-molecular resources in order to facilitate access to resources as well as facilities and to support high quality bio-molecular and medical research.

Although BBMRI is not focusing on intellectual developmental disability bio-banking, it includes several 
relevant bio-banks, such as the cell lines and DNA bank of Rett syndrome and other X-linked mental retardation (biobankUNISI) or the Galliera Genetic Bank: DNA, cell and tissue bank (GGB).

BBMRI-ERIC is expected to increase the efficacy and excellence of European biomedical research by facilitating access to quality human health/diseaserelevant biological resources, including associated data in an efficient and ethically and legally compliant manner. BBMRI-ERIC will also help reduce the fragmentation in this area by contributing to the harmonisation of procedures and implementation of common standards. For countries with less developed bio-banking communities BBMRI-ERIC will also play a role in capacity-building.

An active collaborator of BBMRI and another relevant example is the EuroBioBank [21], a network of bio-banks in Europe that has been supported from 2003 by the European Union over several Framework Programmes for Research and Technological Development. EuroBioBank is providing human DNA, cell and tissue samples as a service to the scientific community conducting research on rare diseases, including a number of diseases characterised by intellectual disability such as Cornelia de Lange syndrome, fragile-X syndrome or Rett syndrome. The added value of European collaborative research on rare diseases is striking. Rare diseases do not affect more than five in ten thousand people in Europe, leading to limited numbers of affected patients of any given disease in any single country and a similarly low number of relevant experts. However, in the EU an estimated 30 million people are suffering from a rare disease. Advancing knowledge on rare diseases thus requires multinational, multidisciplinary approaches, underpinned by adequate research infrastructures. EuroBioBank not only helps researchers to locate relevant samples, it also promotes best practice guidelines for bio-banking activities and helps disseminate knowledge and know-how to the scientific community through training courses.

\section{Training Researchers}

Training researchers has been a long-standing priority for the European Union, with the support offered through the Marie Skłodowska-Curie actions. These actions are meant to provide excellent and innovative research training as well as attractive career and working conditions, and knowledge-exchange opportunities through cross-border and cross-sector mobility of researchers to best prepare them to face current and future societal challenges [8].

Some 45 FP7 Marie Skłodowska-Curie grants [22] supported research fellows in their work around intellectual disability. Examples include the study of large-scale protein expression profiling of genes implicated in cognitive disorders, including disorders characterized by intellectual disability, and the dissection of their role in neural tissue development and plasticity; or the clarification of cellular mechanisms of Rett syndrome in animal models or testing the efficacy of new therapies for Rett syndrome.

An important programme in the intellectual disability area is co-funded by FP7. The Assistive Technologies in Autism and Intellectual Disability (ASSISTID) COFUND Training \& Mobility Programme [23] is aimed to provide Europe with highly skilled professionals in assistive technologies and behavioural sciences applied to autism and intellectual disability. The programme will equip postdoctoral researchers with multidisciplinary skills to enable them assume leadership roles in intellectual disability research and with the services, which provide for the education, social inclusion and employment of people with autism or an intellectual disability. By promoting research into the development and application of assistive technologies, ASSISTID will ultimately enhance the quality of life of people with intellectual disabilities and autism, their carers and families. Combating stigma and reinforcing the interdisciplinary approach to brain research were amongst the key recommendations that came out of the discussions held during the Month of the Brain in May 2013 [24], organised at the initiative of the European Commission, with a view to showcasing European Union efforts in the field of brain research.

\section{HORIZON 2020: RESEARCH ON INTELLECTUAL DISABILITY}

Challenges faced by people with mental disabilities, their families and carers call for excellent research to better understand the problems and find the right answers to be delivered at the right time. Top notch innovation is required in Europe to ensure a broader uptake of technological, organisational and social innovations that can empower people with intellectual disabilities to live active and independent lives, and to achieve their potential.

Horizon 2020, the European Union Framework Programme for Research and Innovation (2014-2020) 
[8] provides challenge-oriented, patient-centred and solution-focused opportunities for research and innovation designed to address such challenges. Horizon 2020, with a total budget of EUR 80 billion, is designed to help bridge the gaps between research, the market and the needs of society.

With the specific objective of improving the lifelong health and well-being of all, work under the EUR 7.5 billion 'Health, demographic change and wellbeing' societal challenge of Horizon 2020 will build on the significant investment in collaborative health research undertaken within FP7. Research and innovation actions supported under this societal challenge, SC1, aim to prevent, detect early, manage, treat and cure disease, disability, frailty and reduced functionality. An improved understanding of health and disease requires close links between fundamental, clinical, epidemiological and socio-economic research. Therefore bio-banks, as fundamental resources to carry out such research, are expected to be an integral part of the funded projects. Research and clinical data linked to those bio-banks could also provide the basis for analysing the value added of interventions, in particular non-drug ones such as special education or specific healthcare programmes, identifying the best and most cost effective practices for consideration by healthcare systems.

The "Personalising health and care" call (the first Horizon 2020 call for proposals under SC1) [9] launched in 2014, aimed to create opportunities for real breakthrough research and radical innovation in response to health challenges that need urgent solutions rather than addressing specific diseases, by supporting the translation of findings into the clinic and other health and care settings to improve health outcomes, reduce health inequalities and to promote active and healthy ageing. In line with the challengedriven approach, the call was broken down into a series of areas that provide, from different angles, opportunities to support research on intellectual disability. Topics such as "Understanding health, ageing and disease: determinants, risk factors and pathways", "New therapies for chronic noncommunicable diseases" or "Understanding common mechanisms of diseases and their relevance in comorbidities" mobilise bio-banking components. The first projects selected from this call for proposals are expected to start during the first quarter of 2015.

Horizon 2020 offers further opportunities to support health research. The public-private partnership approach of the Innovative Medicines Initiative 2 [25] has been reinforced. With an EU contribution through Horizon 2020 of up to EUR 1.638 billion and a similar contribution from industry, for a global budget of EUR 3.3 billion, IMI2 aims to develop breakthrough, next generation vaccines, medicines and treatments to tackle Europe's growing health challenges, while securing the international competitiveness of Europe's industry. With a wider scope than its predecessor and involving additional industry participants from the life sciences sector, IMI2 will cover the entire medical research and innovation value chain. Its strategic research agenda identifies mental disorders as a priority to be addressed in the years to come. A better understanding of the disease biology and the development of potential biomarkers are key to increasing rates of diagnosis and treatment success, and to developing more targeted medicines. Such research will have its roots firmly embedded in biobanking.

Furthermore, the Excellent Science priority [26] of Horizon 2020 offers opportunities to support frontier research through the European Research Council (ERC), building on over 20 FP7 ERC grants in the area of intellectual disability, the training and career development of young and experienced researchers via the Marie Skłodowska-Curie actions, and worldclass infrastructures of high European added value. The Industrial Leadership priority [27] will help businesses become more innovative, efficient and competitive. For instance, it will strongly support innovation in small and medium-sized enterprises (SMEs) via a dedicated SME instrument that addresses the financing needs of SMEs in implementing high-risk and high-potential innovation ideas. A first call for proposals in this area was dedicated to clinical research for the validation of biomarkers and/or diagnostic medical devices.

Horizon 2020 hence offers the right tools to develop new solutions for the benefit of all those in need, including mentally disabled patients, their families, their carers and healthcare systems.

\section{REFERENCES}

[1] Gustavsson A, Svensson M, Jacobi F, Allgulander C, Alonso $\mathrm{J}$, Beghi $\mathrm{E}$, et al. Cost of disorders of the brain in Europe 2010. Eur Neuropsychopharmacol 2011; 21(10): 718-79.

http://dx.doi.org/10.1016/j.euroneuro.2011.08.008

[2] Maulik PK, Mascarenhas MN, Mathers CD, Dua T, Saxena $\mathrm{S}$. Prevalence of intellectual disability: a meta-analysis of population-based studies. Res Dev Disabil 2011; 32(2): 41936.

http://dx.doi.org/10.1016/..ridd.2010.12.018 
[3] Wittchen HU, Jacobi F, Rehm J, Gustavsson A, Svensson M, Jönsson $\mathrm{B}$, et al. The size and burden of mental disorders and other disorders of the brain in Europe 2010. Eur Neuropsychopharmacol 2011; 21(9): 655-79.

http://dx.doi.org/10.1016/j.euroneuro.2011.07.018

[4] TACTICS FP7 project, grant agreement number 278948, http://www.tactics-project.eu/ (accessed on 02 February 2015)

[5] Ramsey JM, Guest PC, Broek JA, Glennon JC, Rommelse $\mathrm{N}$, Franke $\mathrm{B}$, et al. Identification of an age-dependent biomarker signature in children and adolescents with autism spectrum disorders. Mol Autism 2013; 4(1): 27. http://dx.doi.org/10.1186/2040-2392-4-27

[6] DENAMIC FP7 project, grant agreement number 282957 , http://www.denamic-project.eu/ (accessed on 02 February 2015)

[7] Llop S, Julvez J, Fernandez-Somoano A, Santa Marina L, Vizcaino E, Iñiguez $C$, et al. Prenatal and postnatal insecticide use and infant neuropsychological development in a multicenter birth cohort study. Environ Int 2013; 59: 175-82.

[8] Regulation (EU) No 1291/2013 of the European Parliament and of the Council of 11 December 2013 establishing Horizon 2020 - the Framework Programme for Research and Innovation (2014-2020) and repealing Decision No 1982/2006/EC

[9] Personalising Health and Care (H2020-PHC-2014-2015; http://ec.europa.eu/research/participants/portal/desktop/en/o pportunities/h2020/master calls.html\#h2020-phc-2014-2015; accessed on 02 February 2015 )

[10] EU-AIMS IMI project, grant agreement number 115300, http://www.imi.europa.eu/content/eu-aims and http://www.euaims.eu/ (accessed on 02 February 2015)

[11] RD-Connect FP7 project, grant agreement number 305444, http://rd-connect.eu/ (accessed on 02 February 2015)

[12] Eleni Zika, Daniele Paci, Tobias Schulte in den Bäumen, et al. Biobanks in Europe: Prospects for Harmonisation and Networking (EUR 24361 EN - 2010; http://ftp.jrc.es/EURdoc/ JRC57831.pdf; accessed on 02 February 2015)

[13] Proposal for a Regulation of the European Parliament and of the Council on the protection of individuals with regard to the processing of personal data and on the free movement of such data (General Data Protection Regulation) (COM(2012) 11 final)

[14] Communication from the Commission: Europe 2020 A strategy for smart, sustainable and inclusive growth (COM(2010) 2020 final); http://ec.europa.eu/europe2020/ index_en.htm; accessed on 17 February 2015
[15] Justice and Home Affairs Council, meeting 04-05 December 2014 (nr 3354; http://www.consilium.europa.eu/en/meetings/ jha/2014/12/04-05/; accessed on 17 February 2015)

[16] European Parliament legislative resolution of 12 March 2014 on the proposal for a regulation of the European Parliament and of the Council on the protection of individuals with regard to the processing of personal data and on the free movement of such data (General Data Protection Regulation) (P7_TA(2014)0212) (http://www.europarl.europa.eu/sides/ getDoc .do?type $=T A \&$ language $=E N \&$ reference $=P 7-T A-2014-$ 0212; accessed on 17 February 2015)

[17] European Strategy Forum on Research Infrastructures http://ec.europa.eu/research/infrastructures/index_en.cfm?pg =esfri (accessed on 17 February 2015)

[18] BBMRI FP7 project, grant agreement number 212111; http://cordis.europa.eu/project/rcn/88365 en.html (accessed on 17 February 2015)

[19] Commission Implementing Decision of 22 November 2013 on setting up the Biobanks and Biomolecular Resources Research Infrastructure Consortium (BBMRI-ERIC) as a European Research Infrastructure Consortium (2013/701/EU)

[20] BBMRI-ERIC http://bbmri-eric.eu/ (accessed on 02 February 2015)

[21] EuroBioBank catalogue (http://www.eurobiobank.org/en/ services/CatalogueHome.html; accessed on 02 February 2015)

[22] EU Marie Skłodowska-Curie actions - Research Fellowship Programme http://ec.europa.eu/research/mariecurieactions/ (accessed on 02 February 2015)

[23] ASSISTID FP7 project, grant agreement number 608728; http://www.assistid.eu/ (accessed on 02 February 2015)

[24] European Month of the Brain (http://ec.europa.eu/research/ conferences/2013/brain-month/index_en.cfm; http://ec.europa.eu/research/conferences/2013/brainmonth/pdf/dublin/dublin_month_brain_conference_report.pdf; accessed on 17 February 2015)

[25] Council Regulation (EU) No 557/2014 of 6 May 2014 Establishing the Innovative Medicines Initiative 2 Joint Undertaking

[26] Horizon 2020 Excellent Science (http://ec.europa.eu/ programmes/horizon2020/en/h2020-section/excellentscience; accessed on 17 February 2015)

[27] Horizon 2020 Industrial Leadership (http://ec.europa.eu/ programmes/horizon2020/en/h2020-section/industrialleadership; accessed on 17 February 2015) 